\title{
Bilateral empyema treated by sequential pleuroscopy
}

\author{
Kostas Archontogeorgis, Anthony Lanfranco*, Andreas Koulelidis, Marios E. Froudarakis \\ Department of Pneumonology, Medical School Democritus University of Thrace, Greece
}

\begin{abstract}
Bilateral empyema associated to infectious pericarditis is an extremely rare, yet life-threatening condition. Pleuroscopy-medical thoracoscopy has proved its efficacy in series of patients with empyema. Yet, all reported cases treated by this technique, concerned patients with pleural infection located to a single hemithorax. We present the case of a 71-year-old man with bilateral empyema treated successfully by sequential pleuroscopy, associated to infectious pericarditis.
\end{abstract}

\section{Introduction}

Empyema thoracis is defined by the presence of frank pus in the pleural cavity and it is distinguished, depending on the presence or absence of loculations, as simple or complex empyema [1]. Thoracentesis, with evaluation of Light's parameters, is the gold standard in the

Corresponding author: Marios E. Froudarakis, MD, PhD, Department of Pneumonology, Medical School, Democritus University of Thrace, 68100 Alexandroupolis, Greece. Tel.: +302551075335 - Fax: +302551030343 E-mail: mfroud@med.duth.gr

Key words: Empyema; pleuroscopy; thoracoscopy; bilateral; pleural effusion; pleural infection; pericarditis.

Conflict of interests: all authors state that they have no conflict to disclose in relation with the manuscript. Also, the patient has signed a written informed consent, giving permission to publish his case.

* Dr. Anthony Lanfranco was fellow during one month (November 2011) of the Interventional Unit of the Department of Pneumonology, University Hospital of Alexandroupolis, Greece. His current affiliation is: Dr. Anthony Lanfranco, Assistant Professor of Medicine, Associate Program Director, Interventional Pulmonology Fellowship, Associate Director for Bronchoscopy Training of the Pulmonary and Critical Care Fellowship, University of Pennsylvania Medical Center, Pulmonary, Allergy \& Critical Care Division, 839 West Gates Building 3400 Spruce Street, Philadelphia, PA 19104, U.S.A.

Received for publication: 1 February 2013

Accepted for publication: 18 April 2013

CCopyright K. Archontogeorgis et al., 2015

Tipografia PI-ME Editrice, Italy

Monaldi Archives for Chest Disease Pulmonary Series 2015; 81:735

doi: 10.4081/monaldi.2015.735

This article is distributed under the terms of the Creative Commons Attribution Noncommercial License (by-nc 4.0) which permits any noncommercial use, distribution, and reproduction in any medium, provided the original author(s) and source are credited. diagnosis of complicated pleural effusions or empyema, whereas thoracoscopy represents the most important alternative to surgical treatment [1]. Bilateral empyema is an uncommon, life-threatening condition, especially when associated to pericarditis. Pleuroscopy (or medical thoracoscopy) is a minimally invasive technique representing the gold standard in the diagnosis of pleural effusion and constitutes an effective therapeutic alternative in case of empyema [2,3]. Yet, its place in the treatment of pleural infection is unclear, since no randomized study exists [3]. Even though pleuroscopy is part of the everyday clinical practice, its use in cases of empyema is limited and in the current literature all reported cases treated by this technique, concerned patients with pleural infection located in a single hemithorax. We present, here, the case of a patient with bilateral empyema complicated with pericarditis, treated with pleuroscopy under local anaesthesia.

\section{Case report}

A 71-year old male, with a history of chronic alcohol abuse and heavy tobacco use ( 60 packs per year), was transferred to our institute presenting acute pericarditis and bilateral pleural effusion. The history of the patient started seven days before the hospitalization with ambulatory unsuccessful treatment for left upper lobe pneumonia. The symptoms worsened three days before the admission with chest pain and shortness of breath. On admission the patient was febrile $\left(39^{\circ} \mathrm{C}\right)$ and dyspnoic with intact mental status. Clinical examination revealed pulsus paradoxus, bilateral ankle edema and a palpable liver. Arterial blood pressure was 135/75 while heart rate was elevated (136 b.p.m.). On cardiac auscultation, heart sounds were irregular and reduced in intensity. ECG showed low voltage with negative T waves. Auscultation of the chest revealed coarse crackles on both sides with decreased breath sounds at bilateral bases. Arterial blood gases demonstrated respiratory insufficiency and oxygen supplementation was administered via nasal cannula. Blood analysis showed leukocytosis at $38.000 / \mathrm{mm} 3$ with neutrophil predominance $(89 \%)$, anemia $(\mathrm{Hb}=9.2 \mathrm{~g} / \mathrm{l})$ and elevated $\mathrm{C}$-reactive protein $(345 \mathrm{U} / \mathrm{dL})$.

Chest x-ray (Figure 1) demonstrated an enlarged heart shadow and bilateral pleural effusions. The echocardiographic examination revealed a large pericardial effusion with diastolic collapse of the right ventricular wall associated to bilateral multiloculated pleural effusion. A pericardial catheter was inserted with drainage of $500 \mathrm{cc}$ of hemorrhagic-purulent fluid. Diagnostic thoracentesis was performed revealing the presence of pus in each hemithorax. The fluid viscosity did not allow measuring parameters such as cells, LDH, or glucose. Sputum and pleural fluid cultures were negative for common microbes and mycobacterium tuberculosis. PCR for mycobacterium tuberculosis complex on both the pericardial and pleural fluid were negative. Cytological examinations of both fluids were also negative. Chest computed tomography confirmed the ultrasound findings.

Antibiotic treatment was initiated with linezolid, moxifloxacin, metronidazole and fluconazole. As the patient's condition was unstable with a class 7 pleural infection according to the Light's classification [1], a decision to perform bilateral thoracoscopy the morning 
after the admission, was taken in order to accurately drain both pleural cavities.

The patient underwent sequential bilateral single-port rigid pleuroscopy, initially on the right followed by the left hemithorax the next day. The procedures were performed with local anesthesia only, while the patient was conscious and breathing spontaneously. Pleuroscopy findings in both sides were compatible with empyema (Figure 2). Multiple loculations and fibrinous adhesions were present, as well as abundant debris in the pleural fluid. Adhesions were lysed using biopsy forceps and the lungs were liberated. The pleural cavity was cleansed and 2000 cc of purulent fluid were aspirated from the right pleural cavity and $1200 \mathrm{cc}$ from the left. Large-bore chest tubes were placed in a sealed drainage system. Pleural tissue specimens were obtained. Cultures of pleural tissue were negative for common microbes or acid-fast bacilli and histological and cytological examination showed findings compatible with acute inflammation.

The patient was then the subject of triple drainage: bilateral chest tubes plus pericardial catheter (Figure 3). Chest tubes were left in place for five days and a total amount of $2190 \mathrm{cc}$ from the left tube and $2600 \mathrm{cc}$ from the right tube of purulent pleural fluid were removed. The pericardial catheter was left in place for three more days and was re-

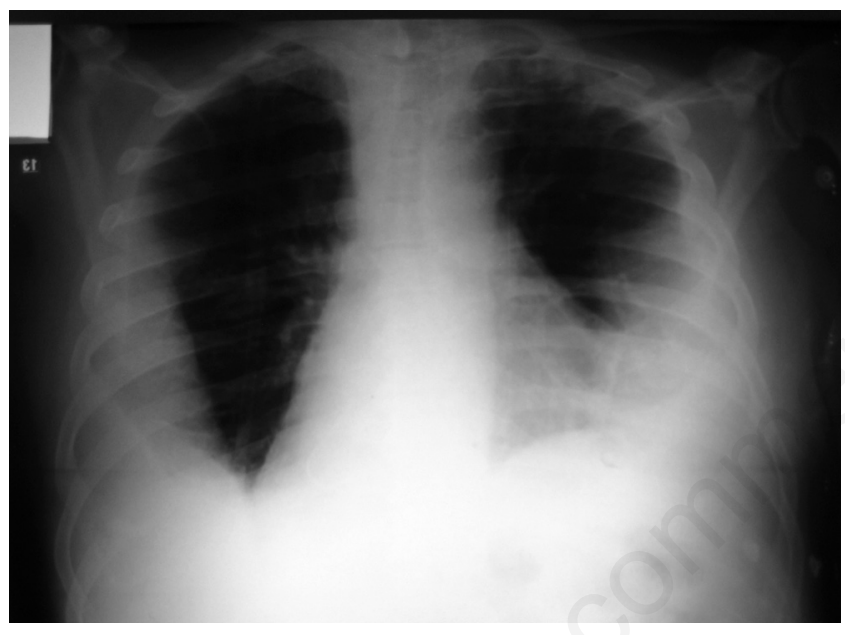

Figure 1. Patient's chest X-ray upon admission showing bilateral pleural effusion.

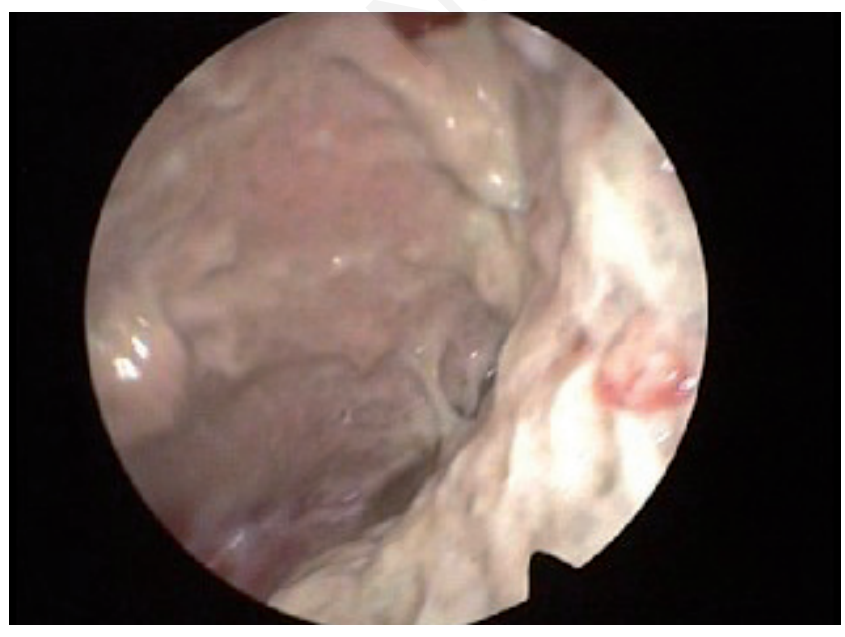

Figure 2. Thoracoscopic view of patient's right pleural cavity, showing a typical empyema. moved after echocardiographic improvement. The patient became afebrile and his clinical conditions were significantly improved during the following days. The patient was discharged eleven days post operatively, with oral antibiotics and was followed in our outpatient clinic with clinical and radiological evaluation. During the follow-up period, his condition continues to improve with significant improvement of his chest X-ray three months after discharged (Figure 4).

\section{Discussions and conclusions}

Pleuroscopy with rigid instruments by single-port access, is a procedure that can be safely accomplished in an endoscopy suite, using local anesthesia in an awake, spontaneously breathing patient [4]. The technique in empyema, allows disruption of adhesions with the creation of a homogeneous pleural cavity, pleural fluid drainage and chest tube placement under direct visual control [5]. Pleural debridement is successfully achieved even though it can be time consuming. In our case, disruption of fibrinous membranes and cleaning of the pleural cavity required approximately 90 minutes for each hemithorax. Our patient treatment by bilareral sequential pleuroscopy was successful, and the patient tolerated very well the operation, despite his critical condition.

Early drainage and an antibiotic treatment, in case of complicated pleural infection, are mandatory. Indeed, it is essential not to delay

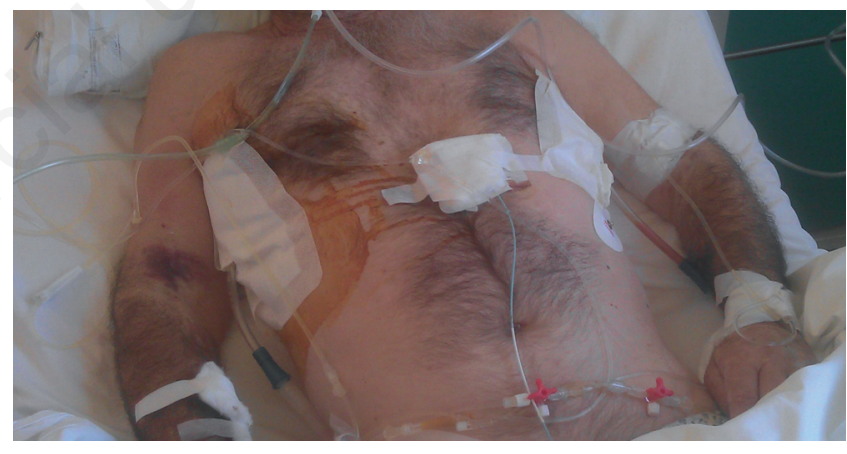

Figure 3. The patient at the first day post-operatively with the pericardial catheter and the bilateral drainage.

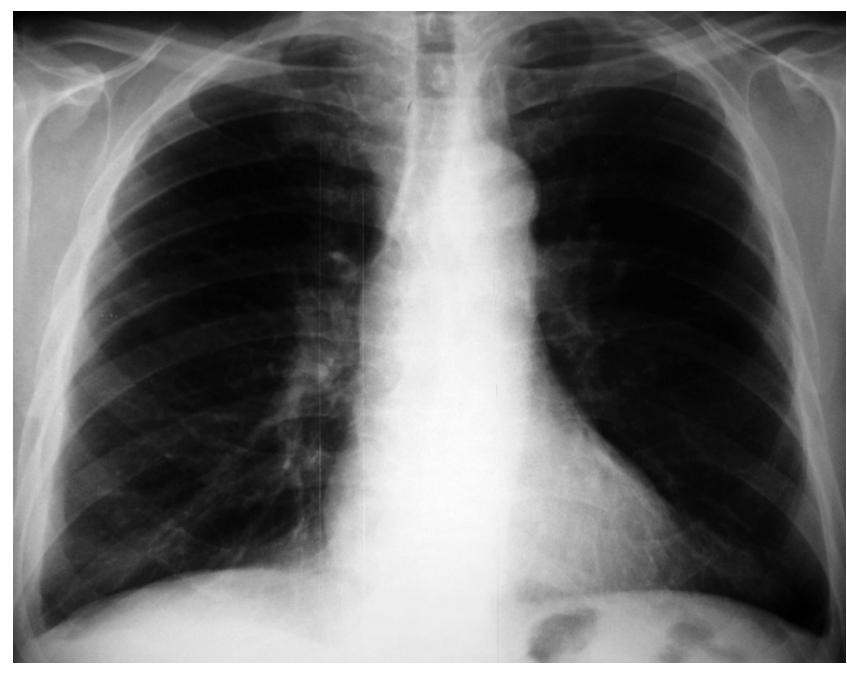

Figure 4. Patient's chest X-ray 3 months after discharge. 
drainage because an effusion that is easy to drain at the beginning can become loculated and difficult to drain over a short period of time [1]. Timing for thoracoscopy is, among others, an unanswered question in the treatment of pleural infection [6]. Our patient presented a class 7 bilateral pleural infection, complicated by septic pericarditis. The indication to perform a minimally invasive treatment is supported by the Light's classification and the recent BTS guidelines, yet it is at the physician's choice according to the patient's clinical status $[1,6]$. Indeed, the two studies reporting results from early intervention with surgical thoracoscopy versus classical treatment in patients with pleural infection, suffer from methodology as they were unblinded, enrolling small number of patients [6] although both showed significant higher efficacy, shorter hospital stay and drainage period, in favor of the thoracoscopy group $[7,8]$. The condition of our patient prompted us to choose the early pleuroscopy approach, to perform an exhaustive debridement and to achieve an immediate complete drainage of both pleural cavities.

Brutsche et al. [5] used chest ultrasound to stratify their patients' with pleural infection, according to the presence or absence of septations. Pleuroscopy was then performed in 127 patients with loculated pleural empyema. Only eight (6\%) out of 127 patients finally required surgical treatment while 119 were successfully treated. Mild complications were observed in 12 (9\%) patients (9 had a short duration air leak and 3 subcutaneous emphysema), while no mortality was recorded. The authors concluded that pleuroscopy can treat successfully and safely the multiloculated pleural infections [5].

Treatment of empyema can be accomplished with an equal efficiency using pleuroscopy and video-assisted thoracoscopy surgery (VATS) [9-11]. Yet, treatement of patients with pleural infection by medical thoracoscopy may save money comparing to surgery, since the technique is less expensive [11,12] and better tolerated than VATS, which requires tracheal intubation [11]. However, its use in this patient population depends very much on local expertise $[11,13]$.

The case of our patient with class 7 bilateral pleural infection, in unstable clinical condition, complicated by pericarditis, shows that pleuroscopy may be an alternative to surgery treatment, in experienced hands for exhaustive debridement and immediate complete drainage of both pleural cavities. However there is a need for large randomized trials to further define the place of this technique in the treatment of pleural infection.

\section{References}

1. Light RW. A new classification of parapneumonic effusions and empyema. Chest 1995;108:299-301.

2. Froudarakis ME. Diagnostic work-up of pleural effusions. Respiration 2008;75:4-13.

3. Froudarakis ME. New challenges in medical thoracoscopy. Respiration 2011;82:197-200.

4. Rodriguez-Panadero F. Medical thoracoscopy. Respiration 2008;76:363-72.

5. Brutsche MH, Tassi GF, Gyorik S, et al. Treatment of sonographically stratified multiloculated thoracic empyema by medical thoracoscopy. Chest 2005;128:3303-9.

6. Davies HE, Davies RJ, Davies CW. Management of pleural infection in adults: British Thoracic Society Pleural Disease Guideline 2010. Thorax 2010;65 Suppl 2:ii41-53.

7. Wait MA, Sharma S, Hohn J, Dal Nogare A. A randomized trial of empyema therapy. Chest 1997;111:1548-51.

8. Bilgin M, Akcali Y, Oguzkaya F. Benefits of early aggressive management of empyema thoracis. ANZ J Surg 2006;76:120-2.

9. Tassi GF, Davies RJ, Noppen M. Advanced techniques in medical thoracoscopy. Eur Respir J 2006;28:1051-9.

10. Kern L, Robert J, Brutsche M. Management of parapneumonic effusion and empyema: medical thoracoscopy and surgical approach. Respiration 2011;82:193-6.

11. Tassi GF, Marchetti GP, Pinelli V, Chiari S. Practical management of pleural empyema. Monaldi Arch Chest Dis 2010;73:124-9.

12. Soler M, Wyser C, Bolliger CT, Perruchoud AP. Treatment of early parapneumonic empyema by medical thoracoscopy. Schweiz Med Wochenschr 1997;127:1748-53.

13. Koegelenberg CFN, Diacon AH, Bolliger CT. Parapneumonic pleural effusion and empyema. Respiration 2008;75:241-50. 